\title{
Erratum
}

\section{Erratum: Grund et al., "Neuropeptide S Activates Paraventricular Oxytocin Neurons to Induce Anxiolysis"}

In the article "Neuropeptide S Activates Paraventricular Oxytocin Neurons to Induce Anxiolysis" by Thomas Grund, Stephanie Goyon, Yuting Li, Marina Eliava, Haikun Liu, Alexandre Charlet, Valery Grinevich, and Inga D. Neumann, which appeared on pages 12214-12225 of the December 13, 2017 issue, the authors discovered errors in their statistical calculations that affect portions of the text and Figures 4 and 5. The authors state: "We discovered several incorrect statistical calculations regarding statistical assessment of outliers according to calculation of lower and upper limits and, consequently, incorrect decisions regarding animals included in or excluded from statistics. Therefore, we (1) included in the statistics the description of this procedure now, (2) consequently included the new datasets in the results of Figure 5, and (3) adapted legends and Figure 5 accordingly. (4) In Figure 4 we found a typo regarding the number of animals per group, without change in statistics and consequently altered Figure 4 indicating proper group sizes now."

A sentence has been added to the Statistics section on page 12225 to indicate processing of behavioral data: "Statistical outliers were calculated using the following formulas: Upper limit $=$ mean $+2 \times$ standard deviation; Lower limit $=$ mean $-2 \times$ standard deviation. In case a value exceeded or fell below the upper or lower limit, respectively, the experimental animal was not included in the statistics."

The Figure 4 legend indicated a group size of rats of $n=5$ or 6 ; the figure and its legend have been updated to reflect the correct group size of $n=4$ or 5 . Figure 5 and its legend have been updated to reflect statistical re-evaluation of behavioral data using upper and lower limit calculations. Specifically, one LDB intracerebroventricular animal was originally excluded without scientific reason and is now included in the statistics; one OF intracerebroventricular Veh/Veh rat meets the lower limit criteria and is now excluded; and one LDB and one OF intra-PVN, Veh/NPS rat meet the upper limit criteria and are now excluded. As a result, some of the statistically significant comparisons shown in Figure 5, especially those from the OF, disappeared without changing the overall message of the results and their interpretation. Also, the $y$-axis now reflects absolute time (in seconds) that animals spent in the lit compartment, rather than percentage of time, resulting in new absolute values.

The section "Pharmacological blockade of OXTR by OXTR-A" has been updated to reflect the statistical reanalysis of the Figure 5 data.

On page 12222, in the Discussion, a new sentence has been added: "Specifically, preinfusion of a selective OXTR-A (Manning et al., 2012) into the cerebral ventricular system prevented the NPS-induced reduction in anxiety levels specifically seen in the LDB, whereas in the OF, the anxiolytic effect of NPS did not reach statistical significance."

Although some statistically significant behavioral effects of pharmacological manipulation of the OXT and NPS systems are lost, these corrections do not affect the conclusions of the paper. The online version has been updated.

DOI: $10.1523 / J N E U R O S C I .0383-20.2020$ 


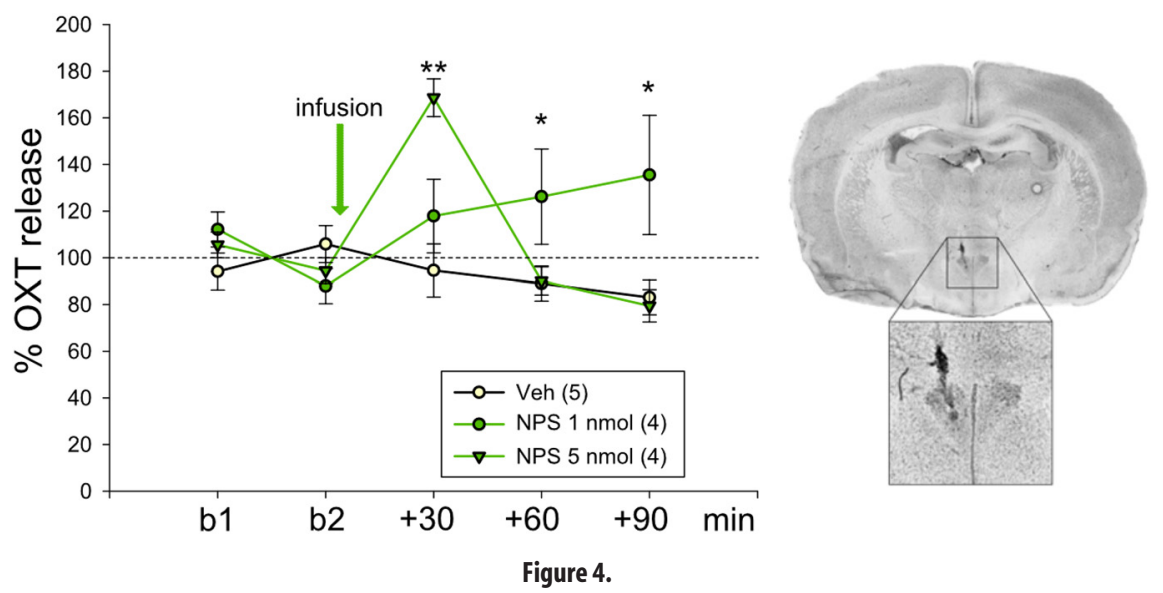

icV $n=8 /$ group, except Veh/NPS in OF: $n=10 \quad \square$ Veh $\square$ NPS
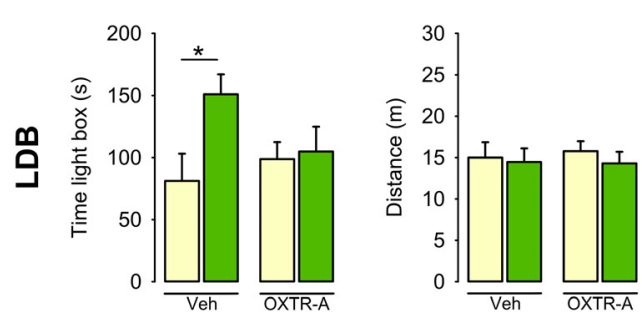

PVN LDB: Veh/Neh (13), Veh/NPS (11), OXTR-AVeh (8), OXTR-A/NPS (9)
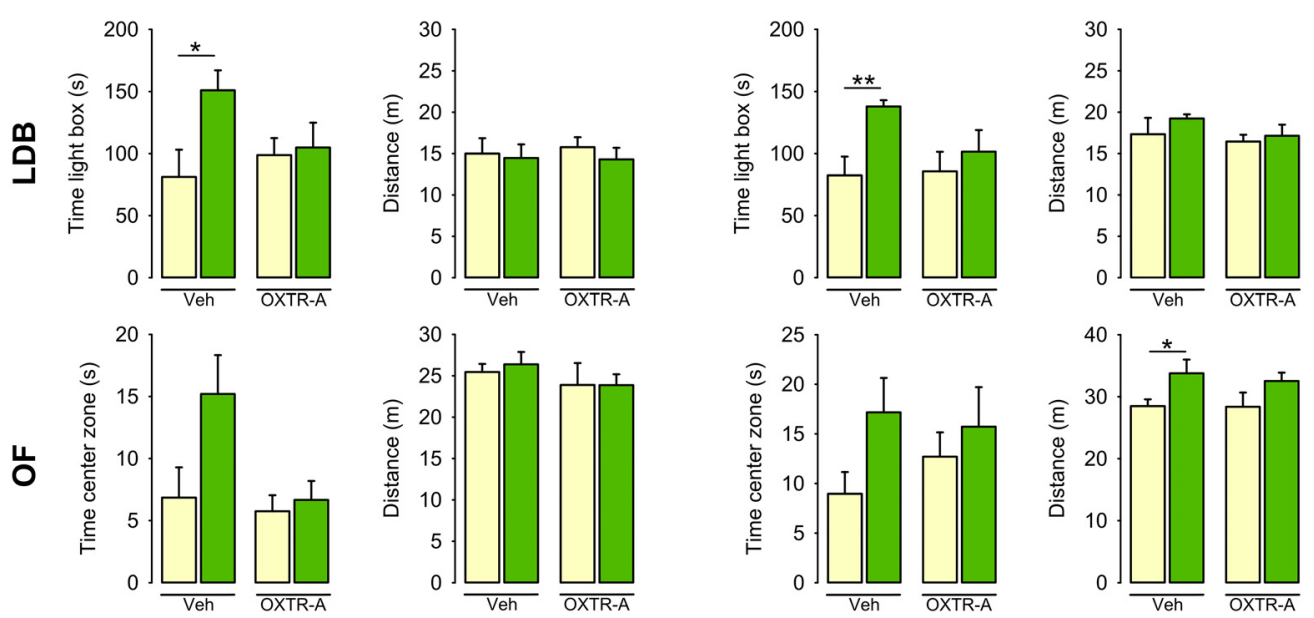

Figure 5. 